\section{Black skies or pale fire?}

SIR - By igniting more than 500 oil wells, Iraq has inflicted an insult to the atmosphere rivalling nature at its worst. The local effects are as appalling as the global effects are trivial. Plumes of soot and pyrotoxins darken the skies over Kuwait and its neighbours as the incandescent flames illuminate scenes of apocalyptic destruction.

The demolition of the water supply to the oilfields makes even master firefighters such as Red Adair despair of extinguishing the wells in less than a year. But even in the absence of water, the appalling soot clouds that threaten the region can be fought - with air.

For so much smoke can only arise from an insufficiency of fire. It is incomplete combustion that is the problem: a stagnating columnar flow of oil, mixed with air only by the turbulence of its burning, is clearly a worst-case as regards air pollution. Can combustion engineering ameliorate it?

The flow rate of each burning well falls into a range familiar to the combustion engineers charged with designing efficient

\section{Degrees of freedom}

SIR - Recent issues of Nature have had articles on the funding for science (or the lack thereof). I would like to address a littleknown but serious problem regarding the funding of scientists who by choice have not attained a degree.

The requirements for receiving research grants from all organizations are dependent on several factors, none more important than whether the applicant has a doctorate or at least is in an academic setting. There are a few of us who do not have a degree but make significant contributions to a chosen field. Where do they get their funding? The lucky few are associated with some institution that may provide funds for their particular research interests (this, however, does not in any way put them on an equal footing with those who have a $\mathrm{PhD}$ ). There are also those who are not affiliated with any institution but who are active in their field purely for the love of the subject. Yet these individuals are severely discriminated against when applying for research grants because they do not possess the required academic papers.

With the economic situation as it stands now, the so-called independent scholar will be even more restricted and less productive. There should be a way to recognize these contributions and provide the necessary funds to keep such people going.

Saskatchewan Museum
$\quad$ of Natural History,
2340 Albert Street,
Regina, S4P 3V7
Canada

TIM T. TOKARYK

NATURE - VOL $350 \cdot 21$ MARCH 1991 thermal power plants fuelled by oil. The 10 -megawatt to half-gigawatt flames at the wellheads in Kuwait are burning only 75-90 per cent of the roughly $1.5-45 \mathrm{~kg}$ per second of gushing oil that feeds them. Adding to each a few tens to hundreds of cubic metres of air per second could eliminate their oxygen deficit and turn them into soot-free oxidizing flames. Better a host of pillars of blue fire ascending than a horde of sullen sources of baleful incandescence and stygian gloom.

The existing wellhead fires are more than sufficiently energetic to drive air entrainment by both convection and, at their bases, the venturi effect. Installing passive combusters built of refractory materials, vortex generators and scaled-up variations on the theme of the Meeker burner or the oilfurnace combuster-can need not await the full restoration of the water lines. And where advection is needed to promote complete combustion, ducted fans and compressors of quite modest power can generate the requisite volumes of air flow - a thrust reckoned in tonnes.

It is time to direct the arsenal of combustion engineering swiftly to deploy whatever it can to reduce the life- and ecosystemthreatening emissions of these flames. Besides, increasing their air supply can reduce their emissivity, cooling the now incandescent ground adjacent to them, thus preparing the way for those who will follow in the long war of attrition that will eventually see them extinguished.

\section{Kennedy Road,}

Cambridge,

Massachusetts 02138, USA

\section{Raman rejoinder}

Sir - In the news item on the Raman Research Institute (RRI) in Bangalore (Nature 349, 732; 1991) your correspondent has rightly stated that $I$ have not commented publicly but would do so in an appropriate forum. Perhaps it is appropriate to comment in Nature if only to clear up distortions, inaccuracies and omissions in the report.

(1) My recommendation to the governing council on Dr C. V. Vishveshwara's contract was not a "sacking" and the abruptness was only apparent. It was certainly not abrupt to the person concerned. Anyone who runs an institute will appreciate the many avenues one goes through in such a case before seeking more drastic remedies. Since things started to go wrong in the mid-1980s, the process has occupied roughly half of Vishveshwara's stay at the RRI.

(2) The international reputation based on his early work was of course the reason why Vishveshwara was invited to set up a school of research in the first place. Your report deals very cursorily with the more relevant question of what was achieved (or not) subsequently. Any visitor to the rest of the astrophysics group at the institute can confirm that the overall atmosphere has been one of friendly interaction, open discussions, participation in seminars and training and encouragement of students and young people. Many colleagues (all junior to Vishveshwara) have contributed to this atmosphere and their work has been recognized by the best form of peer review - invitations to talk at international meetings. If Vishveshwara had measured up to these standards, there would have been no need for me to make a negative recommendation to the council about his contract.

(3) The case was by no means "all set to be closed" when the deciding authority (the governing council) took it up. Three meetings were held and a committee was appointed which submitted a unanimous report. An important part of this report was the stress on building a new school in relativity and gravitation as the old one did not take off.

(4) No restriction on collaboration or the use of institute facilities existed before, during or after the council resolution. The reference to personal research, which has been twisted to imply the opposite in your report, simply refers to the council's perception confirming my belief that Vishveshwara's earlier role as the nucleus of a group should come to an end. The decision I took to shift his office was a natural consequence of his changed role and has been blown up out of all proportion. The new office is a pleasant one, in the same building where I work. More research activity will shift there in the future as and when the need for space arises.

(5) The fact that the unanimous resolution of the council overruled my earlier recommendation and that the trust further tempered the contract and my executive actions clearly show the system of due process and checks and balances at work. This is very far from the image of the institute's functioning conveyed in your report.

(6) A good part of the report covers opinions of various individuals and is thus selective though factual.

(7) The institute's main funding agency, the Department of Science and Technology (represented on its council) is needlessly criticized as being ostrich-like. In fact, its stand reflects its own independent assessment of the role and limits of a funding agency.

I am confident that RRI will continue to be judged by the quality of its atmosphere and research output and will not be found wanting. My colleagues who have worked to build up and maintain this quality would like nothing better than to be allowed to return to their task, without disturbance from ill-informed outcry.

V. RADHAKRISHNAN

Raman Research Institute,

C.V. Raman Avenue,

Bangalore 560 080, India 